\title{
PARADIPLOMACIA Y REGIONALISMO EN SITUACIÓN DE RELACIONES POLÍTICAS EN CONFLICTO: El CASO DE ChILE Y Bolivia*
}

\author{
Paradiplomacy and Regionalism in the Context of Conflicting \\ Political Relations: The Case of Chile and Bolivia
}

\author{
RAÚL BERNAL MEZA \\ Universidad Arturo Prat / Universidad Nacional del Centro de la Provincia de Buenos Aires
}

\begin{abstract}
RESUMEN
El artículo aborda el "regionalismo" y la "paradiplomacia" y la relación entre ambas prácticas de las relaciones internacionales contemporáneas, con el objetivo de estudiar su aplicación a las relaciones entre Chile y Bolivia. Se analiza la evolución teórica y práctica de estos conceptos para su instrumentación en situación de países que no tienen relaciones diplomáticas y derivar elementos que pudieran contribuir a modificar ese estado de situación. Expone los debates teóricos y estudios de casos actuales sobre regiones, regionalismo y paradiplomacia, poniendo atención a su utilización en situaciones de conflicto político, para, posteriormente, aplicar estos conceptos a la situación de las relaciones entre Bolivia y Chile. Se concluye que, debido a las características del Estado "plurinacional" boliviano y las autonomías, podrían modificarse las formas tradicionales en que se relacionan los Estados soberanos y, en esta perspectiva, nuevas concepciones de paradiplomacia podrían ser consideradas en el futuro. De igual manera podrá ocurrir con el "regionalismo" derivado de las formas que adquiera la articulación externa de las regiones.
\end{abstract}

Palabras clave: Regionalismo, paradiplomacia, relaciones internacionales, Bolivia, Chile.

\begin{abstract}
The article discusses "regionalism" and "para-diplomacy", as well as the relationship between these two practices of international relations, and explores possible applications of such practices to the relations between Chile and Bolivia. Theoretical and practical developments and experiences in contemporary international relations are analyzed, to evaluate their prospects in the situation of two countries with no diplomatic relations, and the possibilities of changing that state of affairs. The text sets out, first, the theoretical debates and current case studies on regions and regionalism; then discusses the theoretical and empirical experiences of paradiplomacy, paying special attention to their use in situations of political conflict, to then apply both practices to the status of relations between Bolivia and Chile. As a result of the transformations in the Bolivian State, concepts such as "region" and "paradiplomacy" could be reformulated in the future. The same could be the case with regionalism, depending upon the way in which regions develop their external articulation.
\end{abstract}

Key words: Regionalism, paradiplomacy, international relations, Bolivia, Chile. 


\section{INTRODUCCIÓN}

La diversificación de intereses y mayor autonomía de las regiones nacionales, sus procesos de vinculación con otras pertenecientes a países distintos y la gestión de los asuntos externos por medio de prácticas de paradiplomacia, representan fenómenos que caracterizan las formas que han adquirido las relaciones internacionales. La historia de la paradiplomacia señala que sus antecedentes se remontan al siglo XIX. Más reciente es la articulación con las regiones para comprender algunos escenarios internacionales, de allí que puedan ser analizadas en conjunto; en particular cuando se pretende abordar procesos complejos en el marco de las relaciones entre regiones y sociedades pertenecientes a países distintos y, especialmente, cuando estos no mantienen relaciones diplomáticas. En efecto, la paradiplomacia desplegada por distintos actores en un contexto de regionalismo transfronterizo puede adquirir una particular significación, fomentando así una dinámica de vinculaciones que estimula la búsqueda de mecanismos y nuevas visiones para superar la ausencia de relaciones diplomáticas.

Hasta el presente, las interpretaciones sobre paradiplomacia se refieren a prácticas de actores que son parte de una unidad Estado-nacional, en el marco de un sistema interestatal y que no sustituyen la "diplomacia" del Estado aplicada -en particular-a las agendas de alta política, aun cuando algunas experiencias europeas de paradiplomacia podrían derivar en el futuro hacia el involucramiento en agendas de "alta política" (Baldersheim y Ogard, 2011).

El caso del Estado Plurinacional de Bolivia, sin embargo, podría ser en el futuro el ejemplo de una paradiplomacia de actores no estatales aplicada a la "alta política" y que eventualmente podría también ejercerse en las relaciones con Chile, país que mantiene la "alta política" en el marco de la diplomacia de un Estado de tradición hegeliana. Esta situación podría darse como consecuencia de los grados de autonomía que van alcanzando las regiones y las comunidades originarias bolivianas en el control de recursos y de temas que caben dentro de agendas internacionales vinculadas con el medioambiente y la integración económica.

Conceptos que se utilizan aquí como regionalismo y paradiplomacia no pueden ser definidos en términos absolutos y definitivos, porque -como la experiencia reciente está señalando en las prácticas de actores no gubernamentales de municipios brasileños y estados provinciales de Argentina- remiten a dinámicas que por su naturaleza están llevando periódicamente a ajustes e incluso a redefiniciones de sus contenidos.

La experiencia africana de gestión de conflictos, escasamente conocida, también podría contribuir a una mayor comprensión de las posibilidades que ofrece la paradiplomacia, en la medida que ella proviene de la región del mundo que más sufre conflictos internacionales y problemas fronterizos; pudiendo servir de ejemplo de casos entre países en desarrollo. 


\section{REGIONES Y PARADIPLOMACIA}

El término "regiones" y su nexo con "región", "regionalización" y "regionalismo", remite a un fenómeno externo e interno a los Estados cuya amplitud es relativamente reciente. ${ }^{1}$ Abarca aspectos que caben en el ámbito de las relaciones internacionales aun cuando algunas experiencias podrían suponerse excluidas del campo internacional, por ejemplo, aquellas que responden a la aplicación del término en su exclusivo ámbito nacional. Sin embargo, todas ellas tienen en común el hecho que han venido desarrollando nuevas prácticas de interrelacionamiento denominadas en general como "paradiplomacia".

Si bien tanto el regionalismo como la paradiplomacia reflejan una dinámica en expansión y de creciente importancia, no hay duda que ellas también expresan una confrontación entre las fuerzas centrípetas de los gobiernos centrales y otros órganos del Estado que procuran mantener bajo su control la gestión de los asuntos externos y las fuerzas centrífugas que empujan a los actores subnacionales a la búsqueda de sus propios vínculos externos abarcando distintas agendas según los países involucrados.

\section{DEBATES TEÓRICOS Y ESTUDIOS DE CASOS ACTUALES SOBRE LAS REGIONES Y EL REGIONALISMO}

La interpretación teórica de la regionalización y / o el regionalismo no tiene un consenso en la comunidad política y tampoco en la científica. No hay abstracción o elaboración teórica que pueda abarcar todos los procesos en curso o que puedan ser inscritos en esta denominación. La explicación es que su término hace referencia a distintas concepciones y a procesos diferentes.

Regionalización o regionalismo, como economía política, implican algún grado de integración económico-comercial, hacia un área geográfica o al mundo. Es también una visión de la política externa donde se concibe una forma alternativa de inserción internacional y también puede conducir a la configuración de un "subsistema" internacional. Generalmente, el término remite a la existencia de grandes territorios fácilmente discernibles -África, Asia, Europa, América del Norte y del Sur-. Más difícil es definir regiones heterogéneas y de límites poco claros como el Caribe o Europa central. Los diferentes criterios para identificar regiones dan lugar a diferentes configuraciones regionales: 1) criterios geográficos (situación continental, subcontinental, archipiélago, etc.), 2) criterios militares/políticos (alianzas u orientaciones ideológicas), 3) criterios económicos (desarrollo económico), 4) criterios transaccionales (volumen y frecuencia de los intercambios); pero pueden existir otros criterios: idioma, religión, cultura, densidad de población y el clima (Couloumbis y Wolfe, 1979).

Políticamente la regionalización expresa el tránsito de "nuevas lealtades". La regulación económico-social está transitando, con velocidades distintas, desde los Estados- 
nacionales a las regiones y bloques transfiriendo a estos la autonomía de la política que antes era puramente estatal. Podría considerarse que esta definición es la más actual y de mayor amplitud. Sin embargo ¿cómo incluir en un mismo término los ejemplos del "regionalismo neoproteccionista" (CAN, Mercosur), el "regionalismo abierto" (Chile), el "regionalismo supranacional" (Unión Europea) o el "regionalismo de "regiones o comunidades" (comunidad catalana, autonomía vasca, regionalismo québéçois o el regionalismo de hecho de la "triple frontera argentino-paraguayo-brasileña")?

La construcción de una región descansa en elementos comunes básicos que facilitan la tarea de la integración. El primero es la proximidad y refiere a que a pesar de que las regiones son, a priori, comunidades más imaginadas que reales (Smouts, 1997), ellas tienden a conformarse en territorios donde existen factores comunes previos. Un segundo elemento son los valores compartidos: democracia, desarrollo socioeconómico, industrialización, transformación productiva regional con equidad entre otros. Finalmente, un elemento más son las estrategias de gobernanza común, por ejemplo vínculos de participación, tanto de actores públicos como privados, en términos de acciones sociopolíticas conjuntas, integrando programas y decisiones de gobierno junto a una adecuada participación de las comunidades (sociales, étnicas, políticas) y de los agentes socioeconómicos en una orientación abajo-arriba (bottom-up) y no solo arriba-abajo (top down), que confieren legitimidad y credibilidad (Bernal y Masera, 2008).

Además de este entorno de carácter internacional, están aquellas experiencias de carácter transfronterizo que no se proyectan más allá de la relación entre dos o más países que comparten fronteras y la aplicación del concepto "región" y "regional" al ámbito interno de los Estados.

El término "región" ha sido también ampliado en su acepción desde el momento en que se han agregado las de "macrorregiones" y "microrregiones", las que caben dentro de los acuerdos para la gobernanza territorial. Las primeras implican acuerdos territoriales más o menos fuertes en cuanto a preservar la soberanía de cada uno de los Estados y se especializan en concentrar sus objetivos en el intercambio económico y político con un alto grado de institucionalización. Las segundas representan una formación de grupos por parte de comunidades con características similares de alta marginalización, aislamiento y deterioro y son, por lo general, áreas con economías de subsistencia sin circuitos de producción y consumo (Valadares, 2010).

Las regiones o provincias utilizan y aprovechan la flexibilidad y la fluidez que derivan de la propia acción del Estado y aprovechan también las coyunturas internacionales y las propias necesidades de los actores subnacionales para desarrollar sus acciones. En el Estado se producen situaciones, o se generan circunstancias, en las que esos actores se ubican, generan espacios, aprovechan fisuras, intersticios, omisiones normativas, facultades no delegadas para modificar, resignar y sustituir al poder ejecutivo nacional (Iglesias, 2010: 23). Las unidades subnacionales pueden ser vistas -y el caso brasileño sería un ejemplo- como nuevos actores, aunque no dispongan de autonomía para negociar, firmar acuerdos o hacerse representar, ya que estos son atributos del Estado nacional. Permanecen fuera del derecho internacional, pero de hecho -no de derecho- 
hacen que participen de modalidades de cooperación y establezcan acuerdos económicos o culturales de manera formal o informal (Vigevani, 2004).

Paradojalmente, pueden ser situaciones en ausencia de un poder central, como otras derivadas de la creciente interdependencia que los mismos Estados impulsan en el sistema internacional, las que permiten el surgimiento de espacios donde las regiones alcanzan una mayor autonomía y/o los actores subnacionales adquieren libertad para concertar y cooperar con otros a través de las fronteras nacionales.

El Consejo de Europa define la región como un conjunto territorial menos vasto que el Estado en el cual los hombres encuentran intereses comunes de naturaleza diversa y en donde, debido a los diferentes lazos geográficos y económicos, a las costumbres y a veces a las lenguas, se ha desarrollado un sentido común de pertenencia a un cierto medio de vida. ${ }^{2}$

Mooney (2001: 76) recurre a las distintas constituciones del derecho comparado en donde se establece la figura de la región y afirma que de ello se desprende que esta, más allá del tratamiento constitucional que se le otorgue, debe contar con los elementos que posee todo Estado: territorio, población y gobierno, también una unidad económica como polo de desarrollo propio deben carecer de un poder constituyente propio y ordinario y gozar de cierta autonomía que no llega a ser como la autonomía que poseen las provincias que integran la federación ${ }^{3}$, pero que supera a la génesis de una entidad municipal (Varela, 2010). No obstante, las oportunidades y los riesgos pueden ser compartidos en la medida en que las "regiones a veces pueden ser un instrumento de la política del Estado, a veces un objeto de la política" (Philippart y Van Cutsem, 1999: 796). Asimismo, "la intervención de las regiones en la escena internacional es tolerada en la medida que ella refuerza los recursos del Estado-nación, y siempre y cuando no afecte esencialmente las prerrogativas de este" (ibíd.).

En el caso sudamericano, por variadas razones que refieren a su historia y geografía de los perfiles diversos de sus sociedades, hoy como ayer y como seguramente ocurrirá mañana, la proyección del desarrollo sudamericano es internacional o no es (Caetano, 2010: 150). Pero las relaciones entre las unidades subnacionales y los poderes centrales pueden no llegar a ser aún complementarias. Vigevani (2004), citando a Kugelmas y Branco (2004), señala que dos fuerzas conviven y combaten en la relación entre el gobierno central y los poderes locales: una tendencia centrípeta que procura retener el poder para el gobierno central permitiendo acciones autónomas de las unidades subnacionales siempre y cuando este continúe regulándolas y controlándolas y otra tendencia, centrífuga, que muestra a las unidades subnacionales en búsqueda de una mayor autonomía política y económica.

A partir de las transformaciones ocurridas en el sistema mundial ha ido ganando terreno en las relaciones de cooperación un nuevo paradigma. Se trata de un modelo que

2 Repport d'activité du Comité pour l'aménagement al du territoire Europeen. Conseil d' Europe. Estrasburgo, 1962. Citado por A. Mooney (2001).

Se trata de las provincias en Estados federales, como la Argentina. 
percibe la cooperación entre las estructuras políticas subnacionales no como asistencia unidireccional de los más ricos hacia los más pobres, sino como el intercambio entre unidades territoriales diferentes que establecen vínculos de mutuo beneficio. Es así que se ha ido gestando lo que algunos han llamado la "paradiplomacia" (Caetano, 2010). Este sería, por tanto, el instrumento a través del cual se ejerce la vinculación entre comunidades pertenecientes a Estados distintos, pero no controladas (o dominadas) por el poder central.

Sin embargo, dado que "la proyección de las regiones más allá de sus fronteras representa una anomalía mayor para el enfoque (realista y) neorrealista" (Philippart y Van Cutsem, 1999), debemos salir del enfoque estado-céntrico para comprender la dinámica internacional de las regiones que se mueven por intereses que caben dentro de la economía política de las relaciones internacionales.

\section{Integración y relaciones fronterizas}

Las regiones fronterizas son subsistemas abiertos con comportamientos institucionales peculiares y con poca atención a la legalidad originaria y que desarrollan a cambio nuevas condiciones pactadas explícita e implícitamente entre los actores locales (Navarrete, 2006). Machado de Oliveira señala que "la línea que divide un Estado de otro, la faja que separa (o une; pero no mezcla) una cultura de otra, conspira contra la organización compacta del territorio. La dimensión de la vida en la frontera es bipolar y multiforme" (p. 12). En este sentido, la frontera es un límite que permanentemente debe ser transpuesto. La frontera es un espacio de rivalidad y convivencia, que articula esta separación simbólica en las manifestaciones de sus pobladores, de manera tal que termina produciéndose una especificidad propia, con su singularidad precisa. "Cada frontera es una frontera" (Navarrete, 2006).

De esta forma, no nos referimos solo a los mecanismos que permitieron, y posibilitan hoy, acciones bilaterales tendientes a la explotación de recursos naturales compartidos, sino a la cooperación y complementación en áreas de frontera, caracterizadas por condiciones similares de aislamiento, lejanía respecto de los grandes centros político-administrativos de decisión, necesidad de autosuficiencia, ampliación de la base productiva, detención de la emigración interna hacia los grandes centros urbanos, satisfacción de servicios (salud, educación, turismo) etc.; todas ellas situaciones deficitarias que pueden superarse gracias a la interrelación de aquellas economías dispersas en dos o más países con características geoeconómicas similares y que, por una vinculación favorecida por la cercanía de la frontera, tienen posibilidades de complementarse en bienes, recursos y servicios (Bernal, 1986: 57) como las que precisamente se dan en la frontera chileno-boliviana.

La experiencia de subregiones como la Cuenca del Plata muestra cómo la consideración de las zonas fronterizas -no como áreas finales de la capacidad de acción individual de los Estados, sino como polos dinámicos de cooperación y complementación fronterizaresultó en un proceso que forjó la experiencia para modelos más profundos de integración como el Mercosur. 
Si bien las regiones nacionales en América Latina tienen una tradición histórica de vinculaciones con sus respectivas regiones limítrofes que se remontan a la Colonia, es a partir de la implementación de acuerdos multilaterales de integración cuando se asiste a un replanteamiento de las relaciones bilaterales regionales y de la presencia de un país en el otro. Las regiones limítrofes fueron aprovechando las condiciones pragmáticas que la lejanía de los centros políticos les otorgaba a despecho de las políticas oficiales centrípetas. A medida que la región internacional alcanza una mayor importancia dentro de los objetivos de política exterior de los países, las unidades subnacionales aportan a las agencias nacionales una especificidad y un conocimiento más profundo de las realidades y potencialidades de las áreas limítrofes. Desde esta perspectiva, las regiones contribuyen a fortalecer la capacidad de integración, cooperación y concertación entre países vecinos, mejorando la viabilidad de los subsistemas regionales-internacionales dentro del sistema internacional. La predominancia de las relaciones de cooperación sobre las de conflicto y la aceptación de la idea que las políticas domésticas, en relación a las áreas limítrofes, tienen ciertamente un efecto en las zonas vecinas, la articulación de intereses entre regiones de países limítrofes como forma de crear interdependencias nacionales y la "nueva diplomacia" latinoamericana, todo ello jugaría roles relevantes en el proceso de articulación de las zonas de frontera (Bernal, 1989).

En relación con experiencias de larga data, como la integración europea, hace 10 años la mayoría de los policy-makers eran entusiastas en cuanto al rol de las regiones, mientras se impulsaba una mayor autonomía respecto del Estado. También había un grupo pequeño que veía al Estado como salvaguarda en un clima económicamente más competitivo. Para este, el rol de las regiones era el de desarrollarse con autonomía limitada bajo el paraguas de un Estado-nación fuerte. A pesar de ello, las perspectivas eran que las relaciones de dependencia provocadas por la integración europea probablemente estimularían la generación de redes transfronterizas. Estudios recientes (Baldersheim and Ogard, 2011) dan cuenta de la importancia que se le asigna a los actores políticos como agentes del desarrollo. Son las políticas las que determinan la política, es decir, la aparición de nuevas políticas de desarrollo regional, impulsadas por las regiones, crean presiones en las estructuras institucionales existentes, generando la necesidad de encontrar nuevos patrones organizacionales que se adecuen mejor a la búsqueda de nuevas acciones.

Sin embargo, la experiencia señala que no todas las regiones están en condiciones de enfrentar el desafío de constituirse en pilares del desarrollo local/regional. Ellas compiten entre sí por inversiones nacionales e internacionales. Asimismo, deben enfrentar la oposición de ciudades cercanas que se resisten a las ideas de regiones poderosas. Muchas ciudades se ven como los verdaderos agentes de desarrollo de sus respectivas regiones $\mathrm{y}$, si bien el contexto nacional marca la elección de políticas específicas, la implementación de las mismas es afectada por las diferencias entre ciudad/región en un mismo país. Cuando estas diferencias coinciden con las ideológicas, llevar a cabo políticas de desarrollo regional es una tarea compleja, lo que se proyecta sobre las regiones fronterizas, condicionando los esfuerzos de sus habitantes. Entonces, la paradiplomacia se transforma en un instrumento, pero también en un desafío político frente al Estado 
central aunque las regiones están comprometidas en los procesos de desarrollo local/ regional, que ellas se involucren en la "alta política" como actores de las relaciones internacionales no es una realidad tan lejana (Baldersheim y Ogard, 2011), sino una situación que profundizaría la práctica paradiplomática.

\section{El contexto transfronterizo en el marco de las relaciones entre Chile y Bolivia}

Ciertamente se trata de un contexto político-diplomático complejo, en el que también está incorporado -por historia- Perú. No se trata de profundizar sobre estas características, sino de tomar conciencia que la frontera siempre tiene un carácter integrador aun cuando los Estados no deseen integrarse. Hay factores que crean condiciones favorables para esa integración fronteriza y para una progresiva complementación económica. El hombre de la frontera es un agente de integración, aunque el tránsito fronterizo no sea legal ni regular (Bernal, 1986). Más aún cuando las comunidades que las habitan están relacionadas históricamente por componentes étnicos, culturales y religiosos.

Desde el punto de vista de las distancias y las características de sus poblaciones, en esa zona las fronteras no dividen culturas específicas, religiones, y menos aún áreas geoeconómicas demasiado diferentes, contrapuestas o mejor vinculadas con otras regiones alternativas. Desde el punto de vista de cada país sí se puede entender una vinculación mayor con el todo nacional, pero no desde la perspectiva de un desarrollo conjunto de las posibilidades que ofrecen la complementación y la cooperación multilateral y fronteriza. Es en este contexto que debe analizarse la práctica de la paradiplomacia, en la medida en que -como consecuencia del pasado histórico- los Estados nacionales mantienen visiones en las cuales la percepción de conflicto sigue dominando por sobre la cooperación.

Como han señalado Ovando y González (2014), existen algunos hitos integracionistas emprendidos por las regiones contiguas de Tarapacá-Arica y Oruro desde 1904 y hasta la década de 1960 en donde se puede observar la tensión entre región y Estado nacional debido al protagonismo de una pluralidad de actores, sociales y políticos, que promovieron y le demandaron al Estado chileno una mayor integración con Bolivia, demanda que derivaba de su propio interés regional (o local).

En el nuevo contexto institucional boliviano establecido por la Constitución que reconoce al país como un "Estado plurinacional" y otorga a las comunidades que lo integran distintos grados de autonomía, se creará un marco político totalmente diferente al de Chile y, en general, al de los países que mantienen la concepción estatal de la tradición moderna occidental, de donde derivarán nuevas formas de articulación paradiplomática.

El proceso de "refundación del Estado" que se vive en Bolivia -una de cuyas características es la condición plurinacional- se expresa en las autonomías indígenas, que es el espacio donde se aplica la transformación del Estado (Rojas, 2009). Cada nación reconocida por la Constitución, que tiene ahora poder de decisión sobre los recursos naturales y su propiedad, toma decisiones y/o influye en el proceso de decisión sobre relaciones económicas internacionales, por lo cual actúa en el ámbito internacional. Así, no se ve 
lejano el momento en que estas comunidades asuman acciones de paradiplomacia y que, al igual que las regiones (como en el caso europeo), se involucren en la "alta política" incidiendo directamente sobre las relaciones bilaterales chileno-bolivianas. Pero otro de los problemas que han surgido durante esta etapa fundacional es el regionalismo que, en la forma que ha emergido, es otra fuente de división y de posible conflicto (Crabtree, 2009: 14). Región en Bolivia es sinónimo de "departamento" y remite a cada una de las nueve unidades administrativas en las que está dividido el país. También se entiende como región a las provincias que forman parte del departamento, muchas de las cuales no se identifican necesariamente con este (Roca, 2009). En esta nueva situación política e institucional el regionalismo adquiere distinta expresión que no corresponde a la chilena.

\section{ANTECEDENTES TEÓRICOS Y EMPÍRICOS DE PARADIPLOMACIA. SU UTILIZACIÓN EN SITUACIONES DE CONFLICTO POLÍTICO}

Experiencias y reflexiones en torno a la praxis de paradiplomacia pueden remontarse al siglo XIX. Si bien el concepto surge hacia el final de la década de 1980, el fenómeno habría ocurrido en otros momentos históricos caracterizados por la expansión de las relaciones económicas internacionales, como fue el fin del siglo XIX hasta la Primera Guerra Mundial. Autores como Flaix (1883)4 , Beulieu (1883) ${ }^{5}$ y Cavalcanti (1900) ${ }^{6}$ llamaban la atención por las acciones de los gobiernos "provinciales" en la esfera internacional, inclusive en el campo de los préstamos externos y los riesgos que eso acarreaba para los respectivos gobiernos centrales en ausencia de supervisión por el poder nacional (Maia y Saraiva, 2012).

Polanyi (1992) puso de relevancia el papel que l' haute finance jugó, como "actor transnacional", en el último cuarto del siglo XIX. Siguiendo esa línea, Maia y Saraiva (2012) rescatan los 40 años de paradiplomacia financiera en Brasil, durante la República Velha, 1890-1930.

En los años recientes la tendencia a la utilización de la paradiplomacia ha variado de un país a otro, pero es más avanzada en los países de mayor desarrollo relativo y más inserción internacional, aunque en todos los lugares se caracteriza por un ordenamiento institucional más bien limitado y parcial, aunque los resultados son independientes del marco jurídico que regula la actividad (Maira, 2010). Sin embargo, la experiencia brasileña señala que la baja institucionalidad tiene incidencia en las acciones, lo que contribuye a su limitación; y si bien una mayor institucionalidad por sí sola no brinda garantías para un acción coherente que fortalezca la actividad de los gobiernos subnacionales, la indefinición jurídica no es estimulante para la paradiplomacia (Vigevani y Prado, 2010).

Contemporáneamente el término fue divulgado por Duchacek (1990), quien abandonó su propia terminología de "microdiplomacia" por reconocer que esta podría ser

Citado por Maia y Saraiva (2012).

Ibíd.

Ibíd. 
considerada despreciativamente y prefirió adoptar la denominación de Soldatos (1990), que fue la que pasó a prevalecer. Duchacek estableció diferencias entre la paradiplomacia transfronteriza, la transregional (sin frontera común) y la global que incluye relaciones con todo el mundo (Maia y Saraiva, 2012).

Los trabajos de Der Derian (1987), Soldatos (1990), Aldecoa y Keating (2000), entre otros, aparecen como los principales referentes en la reflexión en torno a las actividades internacionales que han implementado las regiones de distintos países bajo el alero de la política exterior de sus gobiernos centrales y a veces de manera independiente e incluso contradiciendo dicha política (Aranda et al., 2010).

Según Maia y Saraiva (2012), Duchacek y Soldatos encontraron el marco teórico adecuado para analizar e interpretar la actividad internacional creciente de los gobiernos no centrales en el llamado "enfoque global transnacional" y en la noción de "interdependencia compleja" descrita por Keohane y Nye, los cuales al desafiar al realismo defendieron una nueva agenda en las relaciones internacionales, caracterizada por un número creciente de temas complejos, sin clara jerarquía o fácil solución y que englobaban todos los niveles de gobierno.

Básicamente, la paradiplomacia puede tener dos orígenes: como resultado de prácticas de grupos sociales, poblacionales, étnicos, religiosos o culturales, en ausencia o debilidad estatal y como resultado de la expansión de las atribuciones y derechos de la ciudadanía; como expresión de la expansión de la sociedad civil. Debe ser entendida por oposición o alternativa a la diplomacia ejercida por los gobiernos nacionales y puede ser definida como el "involucramiento de los gobiernos subestatales en las relaciones internacionales" (Caetano, 2010).

La definición por oposición también da la pauta sobre cómo la paradiplomacia debe operar. Haciendo referencia a otro autor -Riordan (2005a)-Zubelzú señala que los actores subnacionales deben aprender de la diplomacia estatal (en su mayoría las lecciones son de carácter negativo: cómo no hacerlo), así como aprovechar las ventajas de ser actores subnacionales: ausencia de bagaje diplomático burocrático, mayor flexibilidad en el diseño de los planes, etc. (Zubelzú, 2010: 191).

Desde los años noventa, la literatura especializada había ampliado el concepto de paradiplomacia para indicar la participación de otros actores estatales diferentes del Estado nacional en la acción internacional. ${ }^{7}$ La creciente presencia de los niveles subnacionales en el escenario externo opera vía contactos formales e informales con entidades públicas o privadas extranjeras en los límites difusos a los cuales cada entidad subnacional está constitucionalmente vinculada.

La paradiplomacia surge en espacios geográficos y/o condiciones en los que el Poder Ejecutivo Nacional o el Poder Central se encuentran ausentes, declinan su presencia activa, no cuentan con los medios y mecanismos para imponer su presencia o los actores 
subnacionales que implementan esa práctica encuentran que resulta un mejor instrumento para alcanzar sus objetivos de carácter regional. La misma pone en control sobre todas las áreas a actores y agendas.

El activismo internacional de los gobiernos subnacionales (municipales y de nivel intermedio) es un fenómeno de alcance universal que está al alza, situación que es propia del contexto de globalización económica y que se ve especialmente favorecida por factores diversos como la descentralización administrativa, democratización política, reivindicaciones nacionalistas regionales o procesos de integración en curso, y aunque actividades identificadas como pertenecientes a su ámbito (cooperación técnica y política internacional, promoción comercial y captación de recursos) ya venían llevándose a cabo de manera más o menos dispersa por los distintos órganos de los gobiernos subnacionales, lo que tuvo lugar en estas últimas dos décadas y especialmente en la última, es un reordenamiento y coordinación de las mismas, acompañados en muchos casos de la elaboración de estrategias de actuación internacional coherentes y a medio o largo plazo, es decir, por "políticas exteriores" en sentido de actividad externa planificada y apoyadas por estructuras administrativas de muy variada índole y composición (Salomón, 2010).

A pesar de ello, hay una importante diferencia en relación a la dimensión o profundidad de la praxis de paradiplomacia. Mientras que existen prácticas de este tipo que buscan ser el germen de un proceso de secesión, en los países latinoamericanos esta tendencia tiene grados diversos de desarrollo, siempre sobre la base del reconocimiento de la unidad nacional y de respeto por el papel que les corresponde al Jefe de Estado y a las cancillerías de cada país (Maira, 2010).

Todas las experiencias de paradiplomacia requieren como paso previo un proceso de descentralización que distribuye el poder entre actores diversos. Sin embargo, hay importantes diferencias entre los distintos niveles subnacionales en relación a su capacidad de acción. Estas diferencias también ocurren por la diversidad de escenarios en los cuales operan, así como por la dimensión de las tareas y responsabilidades que ellas mismas asumen o aspiran a llevar adelante.

En relación a las acciones, estas son de diverso tipo. El primero es el de acciones propias -sean estas producto de una iniciativa o de una oportunidad ofrecida desde el exteriorque surgen como acciones generadas o efectivizadas por la provincia sin intervención directa del gobierno nacional ni del sector privado (cámaras, asociaciones, empresas). Las acciones propias pueden realizarse de manera individual en conjunto con otras provincias e incluso originadas en entidades subnacionales de diferentes países. Otro ejemplo son las acciones concertadas con las ciudades y municipios para contribuir a mejorar su desarrollo. Esta variante puede inscribirse en la categoría de acciones coordinadas que, en lugar de estar coprotagonizadas por el Estado-nación y por una o varias provincias, están organizadas por los niveles subnacionales provincial y municipal (Zubelzú, 2010).

Las experiencias de paradiplomacia, entendidas no solo como mecanismo alternativo a la diplomacia estatal, sino como una forma adicional, complementaria o diferenciada para la solución de crisis y conflictos, han evidenciado formas novedosas y originales, 
como se desprende de la utilización de recursos étnicos, culturales, lingüísticos, de género y otros, los que pueden servir como experiencias a considerar ${ }^{8}$ y porque se aplican a agendas de "alta política". 9

\title{
V. ALGUNAS EXPERIENCIAS DE PARADIPLOMACIA EN REGIONES DE PAÍSES EN DESARROLLO: AMÉRICA DEL SUR Y ÁFRICA
}

\begin{abstract}
América del Sur
El accionar de las regiones y sus acciones de "paradiplomacia" reflejan los cambios en la gestión de la política exterior y la participación de nuevos actores. Acompañadas de la expansión de los estudios universitarios dedicados al ámbito internacional, en los casos de Argentina y Brasil, por ejemplo, se ha advertido cómo ha crecido el número de administraciones municipales, provinciales y estaduales que cuentan con oficinas de asuntos internacionales a cargo de profesionales de la especialidad.

Según Salomón (2010: 238), en 1995 al comienzo de la gestión de F.H. Cardoso, los diplomáticos brasileños introdujeron en su discurso el concepto de "diplomacia federativa" (equivalente a paradiplomacia), con el que se pasó a dar legitimidad a la actuación internacional de los gobiernos subnacionales brasileños en su búsqueda de oportunidades de negocios y de cooperación. Según las aclaraciones de los diplomáticos responsables de desarrollar el concepto, por "diplomacia federativa" se entiende no solo el impulso a la actuación internacional a título individual de los gobiernos subnacionales brasileños, sino también su participación -canalizando demandas de gobiernos no centrales- en el proceso decisorio de la política exterior central aunque, según la autora, esa última dimensión se mantiene aún en el plano de la retórica.
\end{abstract}

A este creciente proceso de expansión de la paradiplomacia respondió la administración central con la creación "de una Asesoría de Relaciones Federativas" (1997) en el Ministerio de Relaciones Exteriores cuyo objetivo era coordinar y apoyar a los gobiernos subnacionales en los procesos de integración del Mercosur y con otros países del continente. Cada vez más estos gobiernos subnacionales participan en la organización y en la conducción de la política externa brasileña. Así, los mecanismos estatales nacionales para dar respuesta al fenómeno de spill over con relación al Estado nacional, se ampliaron bajo el gobierno del presidente Lula da Silva con la creación de la Asesoría de Cooperación Internacional Federativa en la Subjefatura de Asuntos Federativos de la Casa Civil, que luego pasó a ser parte de la nueva Secretaría de Coordinación Política y Asuntos Institucionales (Vigevani, 2004).

Es en este sentido que incorporamos las experiencias africanas. Cfr. Koné (2011).

Según el paradigma realista de las relaciones internacionales, la agenda externa de los Estados se divide en asuntos de "alta política", que son los vinculados a las relaciones político-diplomáticas y las militar-estratégicas; en tanto los asuntos de "baja política" corresponden a los temas económicos, sociales, culturales, deportivos, etcétera. 
En el caso chileno, todas las regiones del país poseen estructuras especializadas para la conducción de sus actividades en el exterior llamadas Unidades Regionales de Asuntos Internacionales (URAI). Incluso, en algunos lugares dichas instancias se han convertido en departamentos, superando la calidad de asesor del Intendente para pasar a ser una estructura orgánica de carácter permanente, siguiendo la tendencia identificada en Argentina y Brasil.

En su evaluación, Aranda (et al.) -citando a Abel Gallardo- señalan que un elemento que limita la institucionalización de las actividades paradiplomáticas emprendidas en Chile es la persistencia de un proceso de descentralización parcial, que ha afectado las capacidades de inserción internacional de las regiones, en la medida en que sus actuaciones en el ámbito exterior no siempre encuentran un marco claro y unánime. En sus conclusiones señalan que las actividades paradiplomáticas emprendidas desde la región de Tarapacá en el marco de su proyección subregional han alcanzado un protagonismo importante. Una de las características constantes de las experiencias estudiadas es la vinculación público-privada que se alcanza entre los actores, conformando un círculo virtuoso: universidad-empresa-sector público, sinergia que permite construir una visión estratégica para el desarrollo de la subregión. Otra característica ha sido que las autoridades homólogas de las regiones y comunas de los países vecinos, tanto privadas como públicas, prefecturas, municipios, gobiernos regionales, cámaras de comercio y turismo, han alcanzado una coordinación importante con avances en la misma tendencia. Las experiencias en esta región limítrofe con Bolivia oscilan entre la paradiplomacia mínima y mayor, es decir, considerando cinco variables: extensión geográfica de la acción internacional, métodos operatorios, intensidad de conflictos con la autoridad central, presencia de liderazgo regional que incentive el regionalismo y su relación con organismos internacionales. Variables que evidencian, en una lógica de menor a mayor complejidad, que a medida que las experiencias recogidas se van acercando a la identitaria o protodiplomacia ${ }^{10}$, van surgiendo mayores fricciones con los actores estatales, se van diversificando los intercambios hasta alcanzar el umbral transnacional, se van generando liderazgos locales que chocan con las lógicas estatales y se van autonomizando los vínculos con organismos internacionales evidenciando la existencia de los fenómenos globales (Aranda et al., 2010: 66). Se trata fundamentalmente de relaciones transfronterizas con Bolivia, Perú y el centro oeste sudamericano y, en menor medida, transregionales.

\section{África: ejemplos de paradiplomacia en ausencia del Leviatán}

En África occidental la paradiplomacia -entendida aquí como formas para enfrentar y resolver las crisis y los conflictos- es respuesta a la ausencia de otros mecanismos (no intervencionistas, de carácter externos) para enfrentarlos. Allí los conflictos revisten 
esencialmente dos formas: conflictos entre Estados o de soberanía y los de naturaleza interna. Según Mbonda (2011), casi todas las crisis políticas en las sociedades multiétnicas de África encuentran sus raíces en la afiliación étnica, lo que vincula dimensiones internas e internacionales.

Por resolución de conflictos o de crisis es necesario comprender exclusivamente desde el punto de vista del derecho internacional su solución, es decir, el hecho de poner fin a una situación litigiosa o que puede devenirla.

Si bien todos los países del mundo han conocido en su historia períodos de conflictos -internos y/o externos- graves, "la cuestión fundamental y genérica es aquella de la posibilidad de una democracia, en Estados sin soberanía ni ciudadanos, con poderes que están siempre fuera de las fronteras geográficas y mentales" (Dissaké, 2011: 99).

De allí que al sur del Sahara se hayan puesto en práctica varias formas de regulación de las diferencias. Se trata de métodos tradicionales o modernos de restablecimiento de la paz, implementados por las propias comunidades que sufren el conflicto y que aplican medidas "diplomáticas" de solución, sin ser instituciones o tener el derecho asignado a ejercerlas; pero que, en ausencia de soberanías estatales y con élites de poder cuestionadas en su legitimidad, se levantan como alternativa.

El número de instrumentos puestos en funcionamiento para prevenir, gestionar o resolver los conflictos, mantener la paz y la seguridad es notable. Son respuestas que rescatan los aspectos ancestrales, originales, de ciertas culturas antiguas; como otras respuestas enraizadas preferentemente en la tradición idealista del perdón o de la reconciliación por vía de la verdad, la justicia, la constitución y la ley, pero también respuestas que ofrecen la filosofía y la ciencia política. De esta forma, se realza la importancia de los aspectos culturales, étnicos y sociales de esas comunidades en el ejercicio de acciones de paradiplomacia.

\section{APLICACIÓN DE AMBAS CATEGORÍAS CONCEPTUALES -REGIÓN Y PARADIPLOMACIA- A LA SITUACIÓN DE LAS RELACIONES ENTRE BOLIVIA Y CHILE}

En un contexto en el que los Estados territorial-nacionales involucrados no mantienen relaciones diplomáticas, la paradiplomacia adquiere dimensiones muy importantes. No obstante, el entorno de las relaciones puede marcar también los límites de esta praxis. "Las dificultades para que un proceso de integración funcione en el plano regional o subregional para un grupo de países se va facilitando si los componentes subnacionales de ese bloque inician programas efectivos de acercamiento y coordinación" (Maira, 2010: 21). Pero este mismo proceso de vinculación gradual puede verse influido de manera negativa por las características que tiene la relación política bilateral a nivel de Estados. En este sentido, las acciones de paradiplomacia llevadas a cabo por actores regionales y comunidades contribuye a crear medidas de confianza mutua entre las poblaciones a ambos lados de la frontera. Sin embargo, también es posible que se generen conflictos con los respectivos gobiernos centrales en la medida que en ellos domine la visión más 
realista. Como señalan Ovando y González (2014), no cabe duda que hubo un momento crítico en que las expresiones paradiplomáticas de las regiones contiguas de Bolivia y Chile entraron en colisión con la diplomacia tradicional. Nos referimos a la primera década del siglo XX, momento en que los Estados-nacionales desechaban la integración territorial a través de líneas férreas entre Iquique y Oruro pese a que ambas continuaron insistiendo en su factibilidad o reemplazaron la demanda por carreteras en las décadas siguientes. Podemos sostener que este hito se repite constantemente con distintos énfasis durante buena parte del siglo XX.

\section{PARADIPLOMACIA BAJO DISTINTAS FORMAS DE ESTADO}

En el caso de Bolivia, el Estado plurinacional rompe con la concepción del Estado-nación como actor unificado y expresión dominante en el campo de las relaciones internacionales que es el modelo chileno. Si los instrumentos conceptuales, teóricos y metodológicos que disponemos para analizar el caso boliviano están todavía por formularse (Santos, 2010), ¿cómo comprender una visión común de paradiplomacia, en la medida que los actores que la ejecutan tienen jerarquías que pueden trascender la visión Estado-céntrica tradicional?

La idea de autogobierno que subyace a la plurinacionalidad tiene muchas simplificaciones: un nuevo tipo de institucionalidad estatal, una nueva organización territorial, el pluralismo jurídico, nuevos criterios de gestión pública, de participación ciudadana, de servicio y de servidores públicos y, al mismo tiempo, el reconocimiento de la plurinacionalidad significa otro proyecto de país, otros fines de la acción estatal y otros tipos de relación entre el Estado y la sociedad (Santos, 2009: 88-89), que son sustancialmente distintas de las características chilenas.

¿Cuáles son los organismos estatales que representan y expresan la política exterior de esa pluralidad de naciones?

La respuesta permite afirmar que existe un espacio para la paradiplomacia a desarrollar frente a esas naciones que constituyen hoy ese "Estado plurinacional", una cuestión que también se observa en el caso ecuatoriano y que muy bien podría proyectarse en un futuro cercano a países como Perú y Paraguay.

Para Chile es el dilema de lo desconocido: ¿cómo se relacionan y negocian el Estadonación de tradición hegeliana con el Estado plurinacional?, puesto que "el reconocimiento de la plurinacionalidad conlleva la noción de autogobierno y autodeterminación" (Santos, 2010: 88) y, de hecho, la plurinacionalidad implica el fin de la homogeneidad institucional del Estado (Santos, 2010: 92). Asimismo, la idea de autogobierno tiene implicaciones institucionales de carácter estatal-internas que necesariamente deberían proyectarse también sobre la política exterior de la misma manera como ya se proyectan en la política interna.

Pero en el sustrato están las relaciones vecinales que han priorizado perspectivas de poder militares y estratégicas que generaron históricamente relaciones conflictivas y de juego de suma cero, y configuraron una visión del otro en términos de amenaza 
(Correa y García, 2013). Resolver los problemas que derivan de situaciones fronterizas en ausencia de relaciones diplomáticas normales puede ser labor de la paradiplomacia, para lo cual debe aplicarse cierta metodología11:

a) ¿Cómo definir, aceptar, desarrollar y reforzar los intereses comunes de los adversarios o socios, que constituyen la base de los acuerdos políticos?

b) ¿Cómo determinar los intereses básicos, es decir, las demandas esenciales y justas de cada lado, cuya satisfacción es necesaria para cualquier arreglo sostenible?

c) ¿Cuáles son los principios éticos del diálogo?

A estas interrogantes debe anteponerse la que remite al actor responsable de dar inicio a la vinculación. Siguiendo lo que señalan Vigevani y Prado (2010), tomando la experiencia del Brasil, la hipótesis es que la acción paradiplomática depende de la iniciativa de los gobernantes al nivel que sea: estadual, provincial o municipal. Pero ¿cómo comprender estas acciones cuando el territorio al que pertenecen los actores está en un proceso de refundación?

\section{DIPLOMACIA Y PARADIPLOMACIA ENTRE CONCEPCIONES ESTATALES DISTINTAS}

Desde Westfalia la diplomacia ha sido el instrumento de relacionamiento entre las unidades estatales en el marco de un sistema integrado por tales entidades. Supuestamente, en el marco de un sistema interestatal, no habría dificultades en la continuidad del tipo de relacionamiento diplomático.

¿Qué es lo que podría ser modificado por la presencia de un Estado plurinacional? En principio, las unidades dentro del sistema se ajustan a una dinámica conducente a la interacción, es decir, un tipo de relacionamiento que les permite realizar las funciones del sistema -en este caso, el interestatal- (Atkins, 1991). Lo que se plantea como hipótesis es que con el ejemplo boliviano estamos en presencia de un proceso de refundación del Estado con la manifestación de la plurinacionalidad de naciones asociadas a la autonomía de las regiones, departamentos y territorios de las naciones y pueblos indígena-originariocampesinos que son la expresión no solo de una concepción distinta del Estado tradicional que desagrega el poder del Leviatán, sino que proyecta una institucionalidad -expresada en la Constitución-, una práctica política -el ejercicio de la autonomía y el control de los recursos naturales- y una concepción del poder -distribuido entre sus naciones- que, necesariamente, deberían modificar la forma consuetudinaria de relacionamiento entre unidades territoriales reconocidas como soberanas dentro del sistema internacional y, en esta perspectiva, nuevas concepciones de paradiplomacia deberían ser consideradas.

11 Si bien ellas están planteadas por Dascal (2011) para situaciones que ocurren en África, las mismas pueden ser aplicadas a otros casos para la solución de conflictos. 
En este contexto, ¿qué concepción de paradiplomacia podemos utilizar para aplicar a las relaciones chileno-bolivianas?

Una de las definiciones de paradiplomacia más difundida es la de Cornago (2000: 56), quien la define como "la participación de los gobiernos no centrales en las relaciones internacionales a través del establecimiento de contactos permanentes o ad hoc con entidades públicas o privadas extranjeras con el propósito de promover diversos aspectos socioeconómicos o culturales, así como cualquier otra dimensión exterior de sus propias competencias constitucionales".

Philippart y Van Cutsem (1999) toman como caso de estudio la experiencia europea y proponen el término relaciones internacionales de las regiones de Europa para comprender la dinámica de la "paradiplomacia" aplicando distintas aproximaciones teóricas: Neorrealismo, Economía Política Internacional, Postmodernismo y los estudios a partir del Federalismo.

Una definición más restringida que pone de relevancia la cesión de atribuciones por parte de la institucionalidad Estado-nacional expresa que "la gestión exterior que desarrollan los actores subestatales, involucrándose en las relaciones internacionales por medio de contactos formales e informales, permanentes o ad hoc, con entidades extranjeras en orden al cumplimiento de objetivos sociales, económicos, políticos o de cualquier otra dimensión externa conforme su propia competencia otorgada por la Constitución del Estado" (Gasol, 2010: 40).

Si bien "Europa occidental es, sin embargo, la única zona donde desestructuración y reestructuración tienen lugar en el marco de un juego de cuatro niveles, teniendo cada uno un peso sustancial: mundial, regional (Unión Europea), nacional y subnacional" (Philippart y Van Cutsem, 1999: 790), donde la paradiplomacia ha traspasado esa barrera que separa la política interna de la política exterior, esta ruptura o fragmentación -y la pérdida relativa del control estatal sobre los actores subnacionales y locales- se da en el marco de la protección que brinda la entidad supranacional denominada "Unión Europea", actor que no tiene similar en el ámbito sudamericano.

Ovando y González (2014) señalan que en las últimas dos décadas ha surgido una corriente crítica sobre la diplomacia que cuestiona la idea de que ella esté reservada solo al Estado y a sus agencias y agentes autorizados (Constantinou, 2013). Esta perspectiva plantea superar la idea de que se trata de un arte "extremadamente formalizado y rígido, dentro una más amplia maquinaria de la política exterior del Estado, completamente aislado de las experiencias sociales reales, y privado de cualquier relevancia política inmediata de nuestra vida diaria" (Cornago, 2013: 7-18). Surge así una idea renovada acerca de la diplomacia que toma en cuenta la subjetividad y el contexto histórico en que opera entendida como el "distanciamiento entre seres humanos por poderes simbólicos y restricciones sociales" (Der Derian, 1987). Esta aproximación nos permite un espacio "para el entendimiento diplomático en las más diversas expresiones de la vida social" (Cornago, 2013: 11), dentro de las cuales destacamos para América del Sur las incipientes diplomacias subestatales o paradiplomacia (Aranda y otros, 2010), incluso las emergentes diplomacias indígenas y de los pueblos (Vargas, 2013). 
En general, las definiciones comparten similares criterios. Sin embargo, como la experiencia ha demostrado que no solo gobiernos no centrales se han involucrado en esta praxis, dichas definiciones han quedado muy restringidas, por lo que preferimos definirla como acciones permanentes u ocasionales, de actores nacionales, públicos o privados, no miembros de estructuras oficiales encargadas de las relaciones internacionales del Estado, dirigidas a otro Estado-nacional o plurinacional con el fin de influir sobre decisiones y conductas de actores nacionales de otro Estado. Esta definición podría expresar la evolución conceptual de la paradiplomacia, a partir de la existencia de una concepción distinta de la unidad Estado-nación tradicional.

\section{CONCLUSIONES}

La paradiplomacia rompe con la clásica distinción entre política interna y política exterior de los Estados. Tanto la acción de vinculación externa de las unidades subnacionales y de las regiones, como también la paradiplomacia, reflejan el cambio de las concepciones de la teoría y la praxis, en relación a la visión Estado-céntrica tradicionalmente dominante. Como señalan Philippart y Van Cutsem (1999), mientras la concepción estado-céntrica mantiene la predominancia del Estado sobre el control de los asuntos externos, son los enfoques de economía política los que se abren a la comprensión de los fenómenos de globalización, transnacionalización y regionalización supranacional con la interacción de nuevos actores no estatales incluyendo las regiones.

En relaciones interestatales dominadas por el paradigma realista, las posibilidades que brindan los ejemplos de soft power, como los de la paradiplomacia, son los que tienden a generar condiciones de mayor interdependencia entre las sociedades involucradas mediante vínculos más laxos o más informales que aquellos que se expresan por medio de los organismos y agencias estatales responsables de las relaciones internacionales del país.

La búsqueda de autonomía se da en alguna medida en razón de los problemas que se presentan objetivamente, pero como sugieren los análisis constructivistas, también influyen en la percepción del mundo los valores de que están imbuidos los actores políticos y agentes económicos (Vigevani, 2004).

La participación internacional y regional de los gobiernos subnacionales gana en importancia y podría irrumpir como una cuestión relevante de la política nacional en diversos países. En casos como Brasil el rol de los estados en lo que se refiere al desarrollo y a la planificación, sugiere que el problema de la inserción internacional o de la absorción de la dinámica internacional, global y regional en las acciones subnacionales, es crecientemente relevante y es un indicador del avance de la integración. La expansión internacional de esos gobiernos locales deriva de su mayor flexibilidad para adaptarse a los cambios, necesidades y demandas de los mercados, la tecnología y la cultura (Vigevani, 2004). Asimismo, la voluntad o el empeño puesto por el gobernante son importantes, porque en contextos de baja institucionalidad regulatoria es él quien puede definir el modelo de acción a desarrollar. 
Aun en países con una sólida tradición estatal, donde las sociedades civiles han aumentado considerablemente sus espacios de autonomía, el involucramiento de las regiones en las relaciones internacionales está en ascenso pasando de la cooperación hacia estadios superiores y no se cree que sea una realidad tan lejana que las regiones se involucren en la "alta política", tal como la experiencia europea estaría demostrando.

En presencia de Estados con fuerte sesgo westfaliano y hegeliano-realista, la paradiplomacia no ha sustituido o llenado un espacio vacío, como sería el caso de otros continentes o regiones del mundo, donde la ausencia del Estado, la inexistencia del Leviatán, la presencia de "Estados fallidos" o la construcción de Estados plurinacionales, ha generado el surgimiento de una paradiplomacia que asume las responsabilidades de resolver las crisis y conflictos intra e inter-Estados, como se advierte, por ejemplo, en África. ${ }^{12}$ Es decir, que mientras en América del Sur, por ejemplo, la paradiplomacia se ocupa de temas vinculados a la "baja política" (cooperación económica, desarrollo regional), en África subsahariana esta se ocupa de la "alta política", y si bien en los países desarrollados de Occidente no se advierten situaciones de crisis y conflictos que amenacen la supervivencia de las sociedades, la perspectiva es que las regiones también comiencen a asumir una paradiplomacia de "alta política".

En Sudamérica la experiencia internacional de las administraciones locales (municipios, provincias, estados) y la formación de regiones -nacionales, fronterizas e internacionalesestá en el origen de la paradiplomacia. No obstante, se advierten diferencias importantes entre los Estados federales y los Estados unitarios. En los primeros, entre los que se cuentan Argentina y Brasil, se advierten mayores niveles de autonomía en la gestión internacional de las subunidades nacionales, aunque es posible también que en estos casos el proceso de integración del Mercosur haya influido positivamente en el sentido de transferir a las sociedades locales mayores cuotas de decisión sobre las formas, vínculos e instrumentos para mejorar su desarrollo, utilizando las ventajas de una mayor inserción internacional.

Respecto del caso chileno, las actividades internacionales de los entes subnacionales no resultan de competencias y funciones constitucionales, sino de dinámicas originadas en la política de inserción internacional del país y la integración con América Latina, en particular, con los vecinos (Fuentes, 2011: 23).

En el caso boliviano, el proceso de expansión internacional de las comunidades que integran el Estado plurinacional se da como consecuencia de la refundación del Estado y derivado de un proceso de confrontación de las nacionalidades con el Estado tradicional. Es el Estado moderno-nación aymará (Rojas, 2009: 71); en tanto "la plurinacionalidad implica el fin de la homogeneidad institucional del Estado" (Santos, 2009: 92). De estas dinámicas y de la gestión del regionalismo surgen o surgirán, inevitablemente, formas de relacionamiento en el marco de la paradiplomacia que deberían ser ajenas a la "diplomacia" ejercida por unidades estatales tradicionales en las cuales el poder del Leviatán no estuviera fragmentado. 
Desde el punto de vista histórico, pese a que por un lado se reconoce un proceso de centralización de la diplomacia hacia actores estrictamente gubernamentales, sobre todo en períodos de mayor conflictividad vecinal, no podemos desconocer la existencia de otros actores sociales presentes en los vínculos entre chilenos y bolivianos, entre ellos las respectivas autoridades regionales, intendentes, delegados presidenciales, empresarios y sindicatos, junto a los comités cívicos que proliferaron en varios hitos clave de las relaciones bilaterales entre los dos países (Ovando y González, 2014).

En cuanto a la paradiplomacia en el marco de estas relaciones, deberían destacarse algunos aspectos particulares de la situación en ausencia de relaciones diplomáticas bilaterales. El primero es el papel que pueden jugar los gobernantes (regionales, locales) en el lanzamiento y continuidad de dicha práctica. El segundo es que el vínculo establecido puede servir como canal de comunicación para instancias superiores de poder que pueden utilizarlo para profundizar un proceso sin la necesidad de involucrase públicamente en una dinámica que podría encontrar al inicio distintos grados de oposición. El tercero se relaciona con la proyección que alcanzarán las autonomías del Estado-plurinacional y cómo estas se insertarán en distintas dinámicas internacionales: desde la región, el regionalismo y la paradiplomacia.

Respondiendo a la pregunta acerca de cómo se pueden llevar los intereses regionales al marco de la dimensión latinoamericana (...), creo que pueden señalarse al respecto cuatro niveles: 1) a través del contacto y la concertación directa entre regiones limítrofes afines con coordinación a nivel de las cancillerías o ministerios del Interior, 2) a nivel de los Ministerios de Relaciones Exteriores manteniendo en dichas agencias representantes regionales ad hoc,3) a nivel internacional directo entre las regiones y el gobierno central de la nación vecina; 4) por medio de acuerdos y tratados bilaterales entre países en donde se establezcan mecanismos para la participación activa de regiones o provincias específicas (Bernal, 1989: 586). Pero no se debe perder de perspectiva el escenario que se viene en relación con el cuestionamiento que la "refundación del Estado" implica para la práctica de la diplomacia tradicional y el espacio de acción que se genera para nuevas dimensiones de la paradiplomacia, que podrían expresar la evolución conceptual de esta, a partir de la existencia de una concepción distinta de la unidad Estado-nación tradicional.

Mientras son las cuestiones vinculadas a la economía política y a la cooperación las fuentes de profundización de la extensión de la paradiplomacia en América del Sur, incluyendo la experiencia chilena, no lo son en el caso boliviano donde las nuevas dimensiones de esta derivan del nuevo marco político e institucional originado por la refundación del Estado.

De esta forma, la evolución de la práctica de la paradiplomacia ejercida por las comunidades bolivianas y las nacionalidades que representan, que son sujetos de la autonomía, podría conducirla, en un futuro cercano y previsible, a agendas de "alta política" y ejercer esa aplicación al relacionamiento con Chile que mantiene la "alta política" en el marco de la diplomacia de un Estado de tradición hegeliana. Un Estado fuerte y centralizado como el chileno podría también tomar como "objetivo" a uno (o varios) de estos sujetos-actores 
bolivianos de autonomía debilitando así la posición estatal central y establecer con ellos la agenda de "alta política" para entender sobre las relaciones entre ambos países.

\section{REFERENCIAS}

Aldecoa, Francisco. 2004. "La paradiplomacia en la Unión Europea: las relaciones exteriores de las regiones". En La paradiplomacia: las relaciones internacionales de los gobiernos locales, editado por S. Rodríguez. México D.F.: Porrúa.

Aldecoa, F. y Keating, M. 2000. Paradiplomacia: las relaciones internacionales de las regiones. Madrid: Marcial Pons: 29-54.

Aranda, Gilberto, et al. 2010. "Experiencias paradiplomáticas en la región de Tarapacá y su proyección subregional". Estudios Internacionales. 165: 33-74.

Atkins, G., Pope.1991. América latina en el sistema político internacional. Buenos Aires: Grupo Editor Latinoamericano.

Baldersheim, H., Vegard H., and Ogard, M. 2011. The rise of the networking region. Surrey: Ashgate.

Beaulieu, Leroy. 1883. L'État modern et ses fonctions, Paris: Guillaumin Cie Libraries.

Bernal-Meza, Raúl. 1986. "Alternativas para una integración fronteriza entre Arica, Tacna y Oruro". Integración Latinoamericana. 118: 57-64.

Bernal-Meza, Raúl. 1989. "El rol de las regiones en la política exterior: Su potencial de articulación con los países limítrofes". Estudios Internacionales. 88: 559-587.

Bernal-Meza Raúl y Masera Gustavo. 2008. "El retorno del Regionalismo: Aspectos políticos y económicos en los procesos de integración regional". Aportes para la Integración Latinoamericana. 18: 1-18.

Caetano, Gerardo. 2010. "Giro político, reinserción internacional y nuevas formas de cooperación entre Europa y América Latina. Gobiernos subnacionales y cooperación descentralizada". En El rol de los gobiernos subnacionales en los procesos de integración regional, editado por C. Gasol Varela y E. Iglesias. Buenos Aires: CARI y CEBRI, pp. 139-186.

Cavalcanti, Amaro. 1900. Regimen Federativo: a República Brazileira. Rio de Janeiro: Imprensa Nacional.

Constantinou, Costas. 2013. "Between Statecraft and Humanism: Diplomacy and Forms of Knowledge", International Studies Review. 15: 141-162.

Cornago, Noé. 2000. "Paradiplomacia". En Las Relaciones Internacionales de las regiones, editado por F. Aldecoa y M. Keating. Barcelona: Marcial Pons.

Cornago, Noé. 2013. “Diplomacy decentralized: subnational politics and the making of sustainable diplomatic couples in Latin America". En Building Sustainable International Couples: Critical Components of a Strategy Towards Peaceful and Constructive Cooperation, editado por B. Vassort-Rousset. Basingstoke: Palgrave.

Correa, Loreto y García, Viviana. 2013. "Turbulencias desde el mar: Chile y Bolivia". Si somos americanos XIII [1]: 93-21.

Couloumbis, Theodore A. y Wolfe James. H. 1979. Introducción a las relaciones internacionales. Buenos Aires: Troquel.

Crabtree, John. 2009. "Una historia de tensiones no resueltas". En Tensiones irresueltas. Bolivia, pasado y presente, editado por J. Crabtree, G. Gray Molina y L. Whitehead. La Paz: Plural, 9-18.

Dascal, Marcelo. 2011. "Tradition de controverse et résolution de conflit". En Médiation et gestion des conflits: Essais sur les fins et les moyens pacifiques de sortie de crise, editado por C. B. Koné. Frankfurt: Peter Lang, pp. 121-138.

De Flaix, Fournier. 1883. Études économiques et financiers. Paris: Guillaumin Cie Libraries.

Der Derian, James. 1987. On Diplomacy: a genealogy of western estrangement. Oxford: Blackwell.

Dissakè, Emmanuel M. 2011. "Penser ou proposer la démocratie en temps de suspicion". En Médiation et gestion des conflits: Essais sur les fins et les moyens pacifiques de sortie de crise, ob. cit., p. 99.

Duchacek, Ivo. 1990. "Perforated Sovereignties: Toward a Typology of New Actors in International Relations". En Federalism and International Relations: the role of subnational units, editado por H. Michelmann and P. Soldatos. Oxford: Claredon Press, pp. 1-32. 
Fuentes, Claudio. 2011. "Paradiplomacia: necesidad de una teoría desde la acción". Fundación Chile 21. 12 [114] http:/ / www.chile21.cl/2011/01/31/paradiplomacia-necesidad-de-una-teora-desde-la-accin/; consultado el 18 de octubre de 2012.

Gasol V., Claudia e Iglesias, Eduardo. 2010. El rol de los gobiernos subnacionales en los procesos de integración regional. Buenos Aires: CARI y CEBRI.

Gasol V., Claudia. 2010. "Paradiplomacia e integración regional en el contexto de la globalización y la democratización. Aspectos constitucionales e internacionales. La experiencia del Foro Consultivo de Municipalidades, Estados, Provincias y Departamentos del Mercosur". En El rol de los gobiernos subnacionales en los procesos de integración regional, ob. cit., pp. 35-136.

Iglesias, Eduardo. 2010. "Introducción. Un encuentro en el foro de las entidades subnacionales en el federalismo y en estados unitarios. Mirada desde la Argentina en el escenario internacional". En El rol de los gobiernos subnacionales en los procesos de integración regional, ob. cit., pp. 21-31.

Kugelmas, Eduardo. y Branco, Marcello. 2004. "Os governos subnacionais e a nova realidade do federalismo". En Governos subnacionais: inserção internacional e integração regional, editado por T. Vigevani y L.E. Wanderley. São Paulo: Editora PUC. pp. 161-188.

Maia, José. N. y Saraiva, José. F. 2012. “A paradiplomacia financeira no Brasil da República Velha, 18901930". Revista Brasileira de Política Internacional, Brasília, 55 [1]: 106-134.

Maira, Luis. 2010. "Introducción". En La Política Internacional Subnacional en América Latina, editado por L. Maira. Buenos Aires: Ediciones del Zorzal, pp. 9-37.

Mbonda, Ernest-Marie. 2011. "Crises politiques et refondation du lien social: quelques pistes philosophiques", En Médiation et gestion des conflits: Essais sur les fins et les moyens pacifiques de sortie de crise, ob. cit., pp. $45-60$.

Midón, Mario. 1998. Derecho de la integración: Aspectos institucionales del Mercosur, Santa Fe: Rubinzal-Culzoni. Mooney, Alfredo. 2001. Derecho público provincial. Córdoba: Advocatus.

Navarrete Margarita. 2006. “Región fronteriza uruguayo-brasilera. Laboratorio social para la integración regional: cooperación e integración transfronteriza". En Diploma en Estudios Internacionales e Integración. Montevideo: Facultad de Ciencias Sociales, Universidad de la República.

Ovando S., Cristián y González M., Sergio. 2014. "La relación bilateral chileno-boliviana a partir de las demandas tarapaqueñas: aproximación teórica desde la paradiplomacia como heterología". En Estudios Internacionales. vol. 46, No 177. http://dx.doi.org/10.5354/0719-3769.2014.30868.

Paquin, Stéphane y Lachapelle, Guy. 2003. “¿Por qué las regiones tienen relaciones internacionales?". En Globalización, Gobernanza e Identidades, editado por F. Morata, G. Lachapelle y S. Paquin. Barcelona:Fundación Charles Pi y Sunyer D’Éstudies Autonómics i Locals.

Philippart, Éric y Van Cutsem, Michaël. 1999. “De l'explication à la prévision: analyse des perspectives en matière de relations internationales des régions d'Europe". En Études Internationales, vol. 30, $\mathrm{N}^{\circ} 4$, pp. 789-808, http://id.erudit.org/iderudit/704090ar

Polanyi, Karl. 1992. La gran transformación. Los orígenes politicos y económicos de nuestro tiempo. México D.F.: FCE.

Riordan, Shaun. 2005. Adiós a la diplomacia. Madrid: Siglo XXI.

Riordan, Shaun. 2005a. "Las relaciones exteriores de Bizkaia: una agenda práctica", ponencia presentada en la jornada "La estrategia exterior de los gobiernos subestatales", Bilbao: Diputación de Bizkaia.

Roca, José. 2009. "Regionalismo, revisitado". En Tensiones irresueltas. Bolivia, pasado y presente, editado por J. Crabtree, G. Gray Molina y L. Whitehead. La Paz: Plural, pp. 71-92.

Rodríguez, Sergio. 2004. La paradiplomacia: las relaciones internacionales de los gobiernos locales. México D.F.:Porrúa.

Rojas P., Ramiro. 2009. Estado, territorialidad y etnias andinas. La Paz: Universidad Mayor de San Andrés.

Salomón, Mónica. (2010), “El Foro Consultivo de Municipios, Estados Federados, Provincias y Departamentos del Mercosur en el contexto de la política exterior brasileña". En El rol de los gobiernos subnacionales en los procesos de integración regional, ob. cit., pp. 233-246.

Santos, Buebaventura de S. 2010. Refundación del Estado en América Latina. La Paz: Plural Editores.

Sepúlveda Leandro. 2001. Construcción regional y desarrollo productivo en la economía de la globalidad. Santiago: CEPAL, Serie Estudios y Perspectivas, $\mathrm{N}^{\circ} 3$.

Smouts, Marie-Claude. 1997. "La région comme nouvelle communauté imaginaire?". En Les paradoxes des régions en Europe, editado por P. Le Gales et C- Lequesne, Christian. Paris: La Découverte. 
Soldatos, Panayotis. 1990. "An Explanatory Framework for the Study of Federal States as Foreign Policy Actors". En Federalism and International Relations, editado por H.J. Michelman y P. Soldados. Oxford: Clarendon Press, pp. 34-53.

Torrijos, Vicente. 2000. "La diplomacia centrífuga. Preámbulo a una política exterior de las regiones". Desafíos. Bogotá, 2: 19-54.

Valadares, Nanci. 2010. "Institucionalização na ausência de empoderamento. O caso do Rio de Janeiro". En El rol de los gobiernos subnacionales en los procesos de integración regional, ob. cit., pp. 219-232.

Vargas, Andrés. 2013. "Reinterpretando la diplomacia de los Pueblos", Departamento de Derecho Internacional Público, Relaciones Internacionales e Historia del Derecho, Universidad del País Vasco, Trabajos y ensayos $\mathrm{N}^{\circ} 17$.

Vigevani, T. 2004. “El marco jurídico e institucional para la gestión internacional de los actores subnacionales gubernamentales en Brasil". Integración \& Comercio. 21: 27-46.

Vigevani, Tullo et al. 2010. "Acciones y problemas de la paradiplomacia en Brasil". En La Política Internacional Subnacional en América Latina, editado por L. Maira. Buenos Aires: Ediciones del Zorzal, pp. 177-207.

Zubelzú, Graciela. 2010. "Algunas reflexiones en torno de las acciones de los actores subnacionales y cooperación descentralizada". En El rol de los gobiernos subnacionales en los procesos de integración regional, ob. cit., pp. 187-194.

Raúl Bernal-Meza es Doctor en sociología por la Universidad Católica Argentina con estudios postdoctorales en las Universidades de Sao Paulo-USP y Brasilia. Master (DEA) en Economía Política de la misma Universidad. Master en Relaciones Internacionales de FLACSO-Programa de Argentina y Diplomado de Ciencias Sociales y Políticas de América Latina por la Universidad de Paris III, Sorbonne-Nouvelle. Actualmente es profesor titular de las Universidades Arturo Prat y Nacional del Centro de la Provincia de Buenos Aires. Profesor de la Universidad de Buenos Aires. E-mail: bernalmeza@hotmail.com 
